\title{
MicroRNA-455-3p functions as a tumor suppressor by targeting eIF4E in prostate cancer
}

\author{
YONGXIANG ZHAO ${ }^{1 *}$, MINGYU YAN $^{2 *}$, YE YUN $^{1}$, JIANGUO ZHANG $^{1}$, RUIMIN ZHANG $^{1}$, \\ YAN LI $^{3}$, XIANGMING WU ${ }^{1}$, QIANG LIU ${ }^{1}$, WEI MIAO ${ }^{1}$ and HAISHAN JIANG ${ }^{1}$ \\ ${ }^{1}$ Department of Urinary Surgery of The Fourth Hospital of Baotou; ${ }^{2}$ The Third Affiliated Hospital, Inner Mongolia \\ Medical University; ${ }^{3}$ Baotou Center for Disease Control and Prevention, Baotou, Inner Mongolia, P.R. China
}

Received August 31, 2016; Accepted January 23, 2017

DOI: $10.3892 /$ or.2017.5502

\begin{abstract}
MicroRNAs (miRNAs) are strongly implicated in various cancers, including prostate cancer. Recently, microRNA-455-3p (miR-455-3p) has been shown to be aberrantly expressed in many tumor tissues, but its functions in tumorigenesis remain unknown. In this study, we investigated the role of miR-455-3p in prostate cancer. We found that miR455-3p is markedly downregulated in prostate cancer cell lines and clinical tumor specimens. Gain-of-function and loss-offunction studies showed that miR-455-3p promotes prostate cancer cell growth both in vitro and in vivo. Bioinformatics analysis and Luciferase reporter assays demonstrated that miR455-3p directly targets and suppresses eIF4E, the rate-limiting factor for cap-dependent translation, which plays important roles in the initiation and progression of prostate cancers. Further studies demonstrated that miR-455-3p inhibits capdependent translation and the proliferation of prostate cancer cells through targeting eIF4E. Taken together, our findings suggest that miR-455-3p functions as a tumor suppressor by directly targeting eIF4E in prostate carcinogenesis and may be used as a potential target for therapeutic intervention in prostate cancer.
\end{abstract}

\section{Introduction}

Prostate cancer is the most prevalent malignancy and the second leading cause of cancer-related mortality among men

Correspondence to: Dr Haishan Jiang, Department of Urinary Surgery of The Fourth Hospital of Baotou, Aogen Road, Qingshan, Baotou, Inner Mongolia 014030, P.R. China

E-mail: doctorljiang@aliyun.com

*Contributed equally

Abbreviations: miRNAs, microRNAs; miR-455-3p, microRNA455-3p; eIF4E, eukaryotic translation initiation factor 4E; 3'-UTR, the 3 '-untranslated region

Key words: microRNA-455-3p, prostate cancer, eIF4E, cap-dependent translation in the United States with estimated 180,890 new cases and 26,120 estimated disease related death in 2016 (1). In China, the incidence rate of prostate cancer shows a steady rise over the past years. In 2015, prostate cancer has become the seventh most common cancer and the tenth leading cause of cancer death in China men with 60,300 estimated new cancer cases and 26,600 estimated cancer deaths (2). Currently, hormone ablation therapy is the most common clinical approach to treat prostate cancer. However, although patients exhibit an initial high response rate to androgen withdrawal, most of them ultimately become resistant to the therapy and develop castration-resistant prostate cancer, for which there is no curative therapeutic strategies available at present (3). Hence, there is an imperative need to elucidate the molecular basis of prostate cancer initiation and progression to develop novel therapies against this disease.

Cap-dependent translation plays important roles in the initiation and progression of many cancers through initiating the translation of many oncogenic mRNAs such as cyclin D1 and c-MYC (4). Cap-dependent translation is controlled by the eIF4F complex, which initiates protein translation by recruiting the $40 \mathrm{~S}$ ribosomal subunit to the $5^{\prime}$ end of mRNA (5). The eIF4F complex contains eIF4A, eIF4G and eIF4E and its activity is mainly regulated by the availability of eIF4E, which is controlled by the phosphorylation of 4E-BP1 and the eIF4E expression level (6). Hypophosphorylated 4E-BP1 prevents the assembly of the eIF4F complex by binding the same residues on eIF4E necessary for the eIF4EeIF4G interaction. Phosphorylation of 4E-BP1 weakens its binding to eIF4E and facilitates the formation of the eIF4F complex (7). Phosphorylation of the 4E-BP1 is mainly regulated by mammalian target of rapamycin complex 1 (mTORC1), the role and mechanism of which in the initiation and progression of prostate cancer has been extensively studied (8). eIF4E has been shown to be overexpressed in prostate cancer tissues and its overexpression is significantly associated with poor patient outcomes (9). Silencing eIF4E expression in prostate cancer cells using an eIF4E-specific antisense oligonucleotide (4EASO) markedly suppressed xenograft tumor growth (10). Although the role of eIF4E in prostate cancer development has been characterized, little is known about the underlying mechanism regulating eIF4E expression in the prostate cancer cells. 
MicroRNAs (miRNAs), a class of small noncoding RNAs (19-25 nucleotides), play a crucial role in the post-transcriptional regulation of gene expression by binding to the 3 '-untranslated region (3'-UTR) of target mRNA, resulting in either translational repression or mRNA degradation (11). miRNAs regulate different biological processes, including cell proliferation, apoptosis, differentiation and metastasis, deregulation of which is implicated in the initiation and progression of cancer (12). Accumulating evidence demonstrates that miRNAs are abnormally regulated in various human cancer types and they can function as oncogenes or tumor suppressors in tumorigenesis (13). In human prostate cancer, oncogenic miRNAs, including miR-21, miR-32 and miR-96, have been shown to be aberrantly overexpressed in tumors and contribute to prostate carcinogenesis (14-16). In contrast, miRNAs, such as miR-185, miR-302a and miR-340, are downregulated in prostate cancer and exert tumor-suppressive roles in the development of prostate cancer (17-19).

miR-455-3p has been reported to be downregulated in many tumor tissues, including hepatocellular carcinoma (20), melanoma (21) and thyroid carcinoma (22). However, the exact role of miR-455-3p in tumorigenesis is still unclear. In this study, we found that miR-455-3p was downregulated in prostate cancer tissues compared with normal prostate tissues. We demonstrated that miR-455-3p suppressed prostate cancer cell proliferation and tumor formation. In addition, we identified eIF4E as the target gene through which miR-455-3p exerts its tumor-suppressive function.

\section{Materials and methods}

Tissue samples. The study protocol was approved by the ethics committee of The Fourth Hospital of Baotou. Prostate cancer tissue samples and normal prostate tissues were collected with informed consent from the participants at the Department of Urinary Surgery of The Fourth Hospital of Baotou. Both normal and prostate cancer samples were confirmed by pathological examination. The tissue samples were snap-frozen in liquid nitrogen and preserved at $-80^{\circ} \mathrm{C}$ until use.

Cells and cell culture. RWPE-1 cells were maintained in keratinocyte serum-free medium (K-SFM, Invitrogen, Carlsbad, CA, USA) supplemented with $50 \mathrm{mg} / \mathrm{ml}$ bovine pituitary extract, $100 \mathrm{U} / \mathrm{ml}$ penicillin and $100 \mathrm{mg} / 1$ streptomycin. 22Rv1, LNCaP, C4-2 and PC-3 and DU145 cells were cultured in RPMI 1640 medium (Life Technologies, Grand Island, NY, USA) supplemented with $10 \%$ fetal bovine serum (FBS, Hyclone, Logan, UT, USA), $100 \mathrm{U} / \mathrm{ml}$ penicillin and $100 \mathrm{mg} / 1$ streptomycin. Cells were maintained in a $37^{\circ} \mathrm{C}$ incubator at $5 \% \mathrm{CO}_{2}$.

To generate cell lines stably overexpressing miR-455-3p, $\mathrm{LNCaP}$ and $\mathrm{PC}-3$ cells were infected with appropriate amounts of Lentiviral particles harboring hsa-miR-455-3p (MIMAT0004784) (GeneChem Co., Shanghai, China), followed by selection with $1 \mu \mathrm{g} / \mathrm{ml}$ puromycin (Sigma-Aldrich, St. Louis, MO, USA) for two weeks.

Quantitative real-time PCR. miRNA from cell lines and frozen tissue samples was extracted using a mirVana miRNA Isolation kit (Ambion, Carlsbad, CA, USA) following the manufacturer's instructions. Expression of miR-455-3p was determined using the PrimeScript miRNA RT-PCR kit (Takara, Shiga, Japan) and was normalized to the U6 small nuclear RNA. The forward primer for miR-455-3p is: 5'-ACA CTCCAGCTGGGGCAGTCCACGGGCATATACAC-3'; the reverse primer for miRNAs is the UnimiRqPCR Primer (Takara). The forward primer for U6 is: 5'-GCGCGTCGTGA AGCGTTC-3'; the reverse primer for U6 is 5'-GTGCAGG GTCCGAGGT-3'.

Total RNA from cell lines was extracted using TRIzol Trizol (Qiagen, Valencia, CA, USA) and reverse-transcribed into cDNA using a cDNA Library Construction kit (Takara, Dalian, China) according to the manufacturer's instructions. RT-PCR was performed using the QuantiTect SYBR Green PCR kit (Qiagen) according to the manufacturer's protocols. The primer sequences were as follows: EIF4E (forward: 5'-TACTAAGAGCGGCTCCACCAC-3', reverse: 5'-TCGATT GCTTGACGCAGTCTCC-3'); CCND1 (forward: 5'-TGAAC TACCTGGACCGCT-3', reverse: 5'-GCCTCTGGCATTTT GGAG-3'); MYC: (forward: 5'-GCTGCTTAGACGCTGG ATTT-3', reverse: 5'-CACCGAGTCGTAGTCGAGGT-3'); $\beta$-actin: (5'-GCACAGAGCCTCGCCTT-3'; 5'-GTTGTCGA CGACGAGCG-3').

Transient transfection of miR-455-3p mimics or inhibitors. Chemically synthesized miR-455-3p mimics and antagomiR455-3p were purchased from RiboBio (Guangzhou, China). miR-455-3p mimics or antagomiR-455-3p was transiently transfected into cells using Lipofectamine RNAiMAX Transfection Reagent (Invitrogen) according to the manufacturer's instructions and subsequent measurements were performed 24 or $48 \mathrm{~h}$ later.

Cell proliferation assays. Transfected LNCaP or PC-3 cells were seeded in 96-well plates at 2000 cells per well. Cell proliferation was measured using Cell Counting Kit-8 (CCK-8) (Dojindo Laboratories, Tokyo, Japan) following the manufacturer's instructions. Absorbance was measured at $450 \mathrm{~nm}$ using a multilabel plate reader. Each experiment was performed with three replicates per sample.

Colony formation assay. Transfected LNCaP or PC-3 cells were seeded in a 6 -well plate at 1000 cells per well containing RPMI-1640/10\% FBS medium. After two weeks, the cells were fixed with methanol for $20 \mathrm{~min}$ and stained with $1 \%$ crystal violet (Sigma-Aldrich) for $20 \mathrm{~min}$. The colony numbers were analyzed using ImageJ (Bethesda, MD, USA). Each experiment was performed with three replicates per sample.

Soft agar assay. The cells were plated in 6-well dish at 5000 cells per well, with a base layer of $0.5 \%$ agarose containing RPMI-1640/10\% FBS medium and a top layer of $0.35 \%$ agarose in the same medium. The cells were allowed to grow for three weeks in a $37^{\circ} \mathrm{C}$ incubator at $5 \% \mathrm{CO}_{2}$ before imaging and counting.

Cloning and Luciferase reporter assay. The full-length wildtype 3'-UTR of eIF4E was amplified by PCR with the cDNA from PC3 cells and cloned into pMIR-Reporter plasmid (Life Technologies). Mutations in miR-455-3p binding site of 3'-UTR 
A

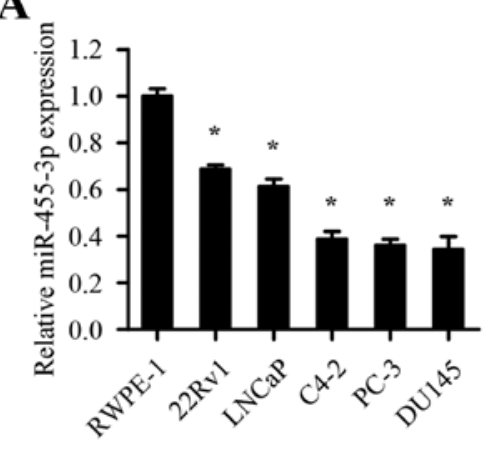

B

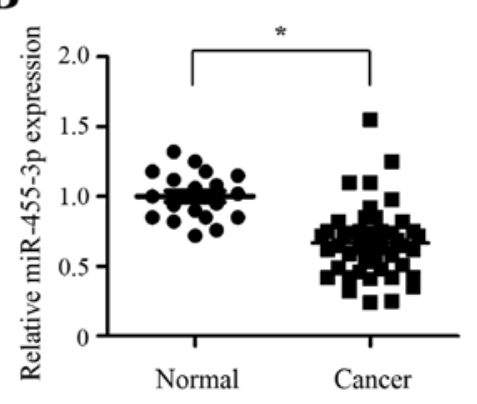

C

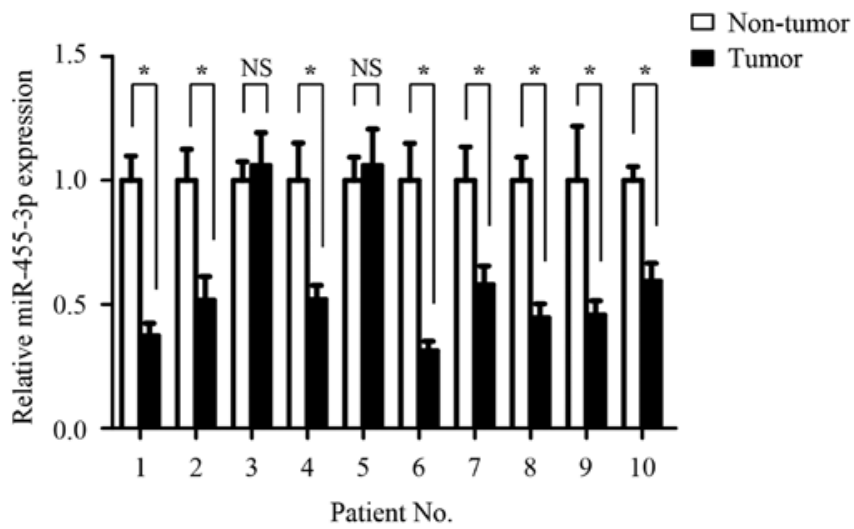

Figure 1. miR-455-3p is significantly downregulated in prostate cancer cell lines and tissues. (A) The qPCR analysis of miR-455-3p for one benign prostate cell line and five prostate cancer cell lines. The expression of miR-455-3p was normalized to U6 snRNA ("P<0.05). (B) The qPCR analysis of miR-455-3p for 18 normal prostate tissues and 47 prostate cancer tissues. The expression of miR-455-3p was normalized to U6 snRNA ("P<0.05). (C) The qPCR analysis of miR-455-3p for 10 paired prostate cancer tissues. The expression of miR-455-3p was normalized to U6 snRNA ( $\mathrm{P}<0.05)$.

of eIF4E was introduced using a QuickChange site-directed Mutagenesis kit (Stratagene, La Jolla, CA, USA) following the manufacturer's instructions. All vectors were confirmed by sequencing.

The cells were seeded into 24 -well plates at $4 \times 10^{4}$ cells per well. The following day, the cells were co-transfected with luciferase reporter plasmids with wild-type 3'-UTR or mutant 3'-UTR of eIF4E, and miR-455-3p mimics or antagomiR455-3p using Lipofectamine 2000 (Invitrogen, New York, NY, USA). At $48 \mathrm{~h}$ after transfection, luciferase activities were determined using the Dual-Luciferase Reporter assay system (Promega, Madison, WI, USA). The pRL-TK vector was used as an internal control. Assays were performed in triplicate and results are presented as means \pm standard deviation (SD).

Western blotting. Cells were lysed in ice-cold RIPA buffer (50 mM Tris- $\mathrm{HCl}, \mathrm{pH} 7.5,150 \mathrm{mM} \mathrm{NaCl}, 1 \%$ sodium deoxycholate, $1 \%$ NP-40, $0.1 \%$ SDS, 1 mM EDTA, 1 mM EGTA) containing protease/phosphatase inhibitor cocktail (Roche, San Francisco, CA, USA). After the measurement of protein concentration using the Pierce BCA protein assay kit (Thermo Fisher Scientific, Waltham, MA, USA), equal amounts of total protein were separated on $10 \%$ SDS-PAGE and transferred to nitrocellulose membranes (Bio-Rad, Richmond, CA, USA). After being blocked with 5\% non-fat milk in Tris-buffered saline containing $0.05 \%$ Tween-20 for $1 \mathrm{~h}$ at room temperature, the members were probed with primary antibodies against eIF4E, cyclin D1 and c-Myc (Cell Signaling Technology,
Beverly, MA, USA) or Tubulin (Santa Cruz Biotechnologies, Dallas, TX, USA) overnight at $4^{\circ} \mathrm{C}$, followed by incubation with HRP-conjugated secondary antibodies for $2 \mathrm{~h}$ at room temperature. Detection was performed using a Super Signal Enhanced Chemiluminescence kit (Pierce Biotechnology, Inc., Rockford, IL, USA).

Cap-dependent translational activity assay. Cap-dependent translational activity in the cells is determined using pcDNA3rLuc-PolioIRES-fLuc plasmid, which directs cap-dependent translation of the Renilla luciferase gene and cap-independent Polio IRES-mediated translation of the firefly gene. LNCaP or PC-3 cells were transiently co-transfected with pcDNA3rLuc-PolioIRES-fLuc plasmid and miR-455-3p mimics or antagomiR-455-3p. At $48 \mathrm{~h}$ after transfection, cells were harvested for luciferase activity assay using the DualLuciferase Reporter assay system (Promega) according to the manufacturer's instructions. Cap-dependent translational activity was measured by calculating the ratio of Renilla/firefly luciferase activity. Assays were performed in triplicate.

In vivo tumorigenesis assay. Male BALB/c nude mice (5-6-week-old) were purchased from the Animal Center for Vitalriver (Beijing, China). To determine the effect of miR455-3p overexpression on the tumor growth, PC3 cells stably expressed miR-455-3p or control cells in $0.1 \mathrm{ml}$ serum-free medium were injected subcutaneously in the right flank of nude mice, respectively. To determine the effect of miR-455-3p 
A

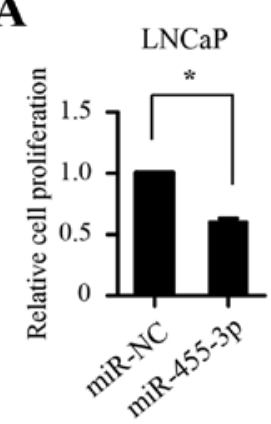

$\mathbf{E}$

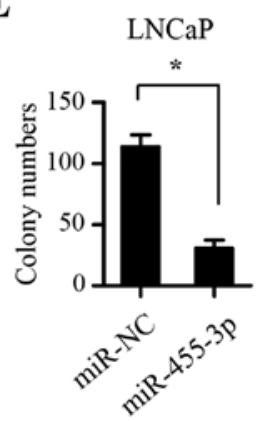

B

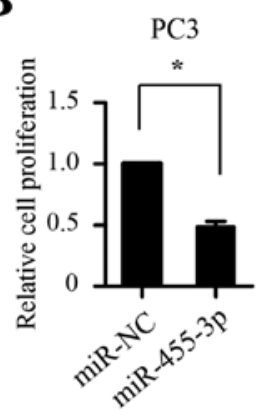

$\mathbf{F}$

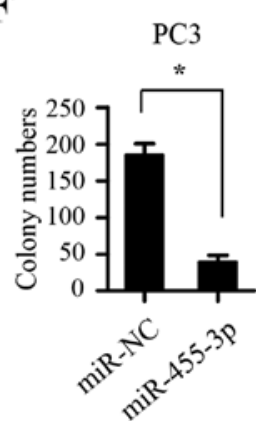

C

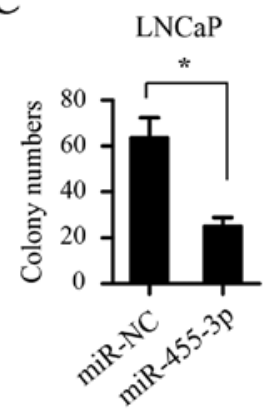

D

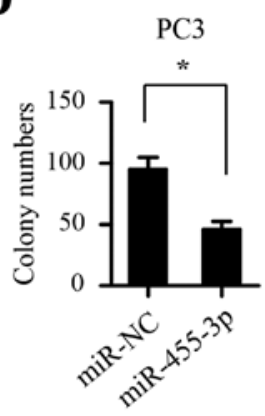

G

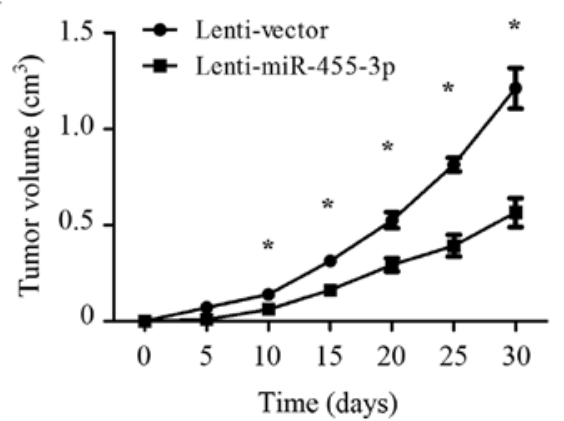

Figure 2. Upregulation of miR-455-3p suppresses prostate cancer cell growth. (A-D) LNCaP and PC-3 cells were transfected with miR-NC or miR-455-3p. CCK-8 assay (A and B) and colony formation assay (C and D) were performed $24 \mathrm{~h}$ after transfection. Results are shown as means \pm SD of triplicate measurements. ${ }^{*} \mathrm{P}<0.05$. (E and F) Anchorage-independent growth ability of LNCaP and PC-3 cells transfected with miR-NC or miR-455-3p was determined using soft agar assay. Results are shown as means \pm SD of triplicate measurements. ${ }^{*} \mathrm{P}<0.05$. (G) $\mathrm{PC}-3$ cells stably overexpressing miR-455-3p and control cells were injected into the flanks of nude mice. Tumor growth measured at the indicated times. ${ }^{*} \mathrm{P}<0.05$.

inhibition on the tumor growth, PC3 cells were injected subcutaneously in the right flank of nude mice. When the average tumor volume had reached approximately $100 \mathrm{~mm}^{3}$, the mice were randomly divided to 2 groups ( $n=6 /$ group) according to body weights and tumor volumes and intratumorally injected antagomir-455-3p (10 nM of antagomir-455-3p diluted in $50 \mu 1$ PBS) or antagomir-NC with two times per week for 3 weeks. Tumor sizes were recorded at indicated times.

Statistical analysis. All data were analyzed with the unpaired Student t-test using SPSS 17.0 software (SPSS, Chicago, IL, USA). The data are presented as mean \pm SD. For all analyses, $\mathrm{P}$-values $<0.05$ were considered to indicate statistically significant differences.

\section{Results}

miR-455-3p is significantly downregulated in prostate cancer cell lines and tissues. To investigate the significance of miR-455-3p in prostate carcinogenesis, the expression of miR-455-3p was measured in one benign prostate cell line (RWPE-1) and five prostate cancer cell lines (22Rv1, LNCaP, C4-2, PC-3 and DU145) using quantitative PCR (qPCR). As shown in Fig. 1A, the expression of miR-455-3p in prostate cancer cell lines was significantly reduced compared with benign prostate cell lines. Next, we investigated whether miR-455-3p is downregulated in clinical prostate cancer tissues. The abundance of miR-455-3p was detected in 65 clinical prostatic tissues, including 18 normal prostate tissues and 47 prostate cancer tissues. As shown in Fig. 1B, the expression of miR-455-3p in prostate cancer tissues was profoundly decreased compared with that in normal prostate tissues, suggesting a potential involvement of miR-455-3p in prostate cancer initiation. To verify the suppression of miR-455-3p in prostate cancer patients, we determined the levels of miR-455-3p in 10 randomly selected prostate cancer samples and matched benign adjacent tissues. Compared with matched benign tissues, 8 of 10 prostate cancer samples (80\%) exhibited marked downregulation of miR-455-3p (Fig. 2C). Taken together, these results from cell lines and clinical specimens suggested that miR-455-3p is aberrantly downregulated in prostate cancer cells and its low expression may be associated with biological process of tumorigenesis.

Upregulation of miR-455-3p suppresses prostate cancer cell growth. The low expression of miR-455-3p in prostate cancer indicates that miR-455-3p may function as tumor suppressor in prostate tumorigenesis. To investigate the role of $\mathrm{miR}$ 455-3p, the miR-455-3p mimics were transfected into LNCaP and PC-3 cells. As shown in Fig. 2A-D, CCK8 and colony formation assays demonstrated that miR-455-3p overexpression significantly inhibited the growth of both LNCaP and PC-3 cells. Soft agar assays showed that ectopic expression of miR-455-3p markedly decreased anchorage-independent growth ability of both LNCaP and PC-3 cells (Fig. 2E and F). To further determine whether miR-455-3p could suppress tumor growth in vivo, PC-3 cells stably overexpressing miR455-3p with a lentiviral vector and control cells were injected into the flanks of nude mice. The results showed that miR455-3p overexpression also markedly decreased the growth 
A

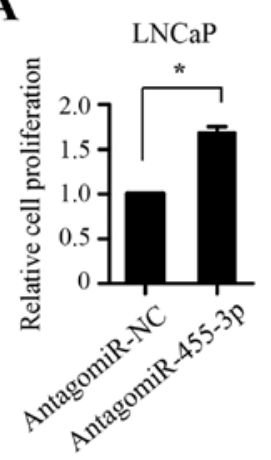

E

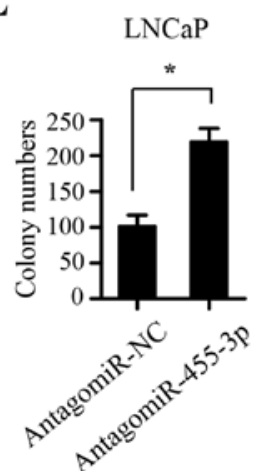

B

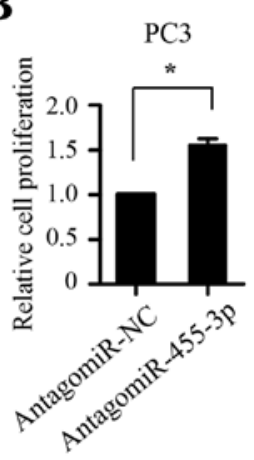

F

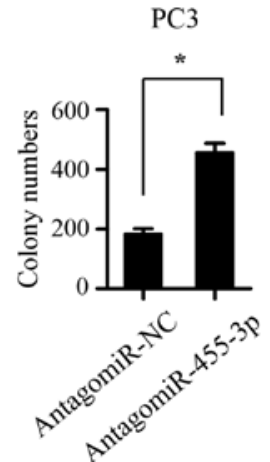

C

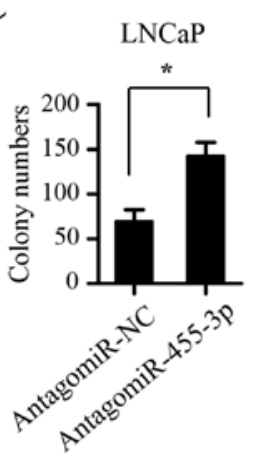

D

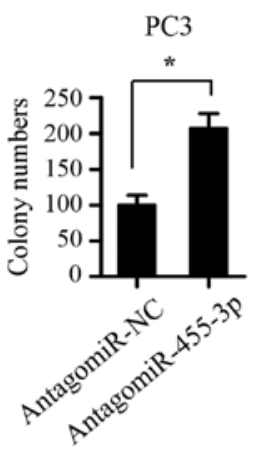

G

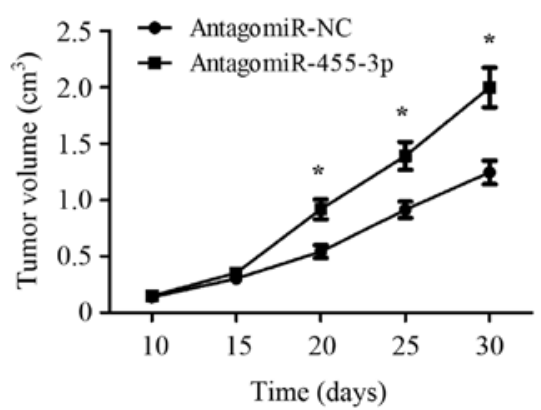

Figure 3. Silencing endogenous miR-455-3p promotes prostate cancer cell growth in vitro and in vivo. (A-D) LNCaP and PC-3 cells were transfected with antagomir-NC or antagomir-455-3p. CCK-8 assay (A and B) and colony formation assay (C and D) were performed $24 \mathrm{~h}$ after transfection. Results are shown as means \pm SD of triplicate measurements. ${ }^{*} \mathrm{P}<0.05$. (E and F) Anchorage-independent growth ability of LNCaP and PC-3 cells transfected antagomir-NC or antagomir-455-3p was detected using soft agar assay. Results are shown as means \pm SD of triplicate measurements. ${ }^{*} \mathrm{P}<0.05$. (G) PC3 cells were injected subcutaneously in the right flank of nude mice. When the average tumor volume had reached approximately $100 \mathrm{~mm}^{3}$, the mice were randomly divided to 2 groups (n=6/group) and intratumorally injected antagomir-455-3por antagomir-NC with two times per week for 3 weeks. Tumor growth measured at the indicated times. ${ }^{*} \mathrm{P}<0.05$.

of subcutaneous xenograft tumors (Fig. 2G). Taken together, these data indicate that miR-455-3p has a suppressor role in prostate cancer cell growth.

Silencing endogenous miR-455-3p promotes prostate cancer cell growth in vitro and in vivo. To confirm the inhibitory role of miR-455-3p in prostate cancer cell growth, we silenced endogenous miR-455-3p using the miRNA antagonist antagomir-455-3p and determined the effect of miR-455-3p downregulation on prostate cancer cell growth. CCK8 and colony formation assays revealed that miR-455-3p inhibition drastically promoted the growth of both LNCaP and PC-3 cells (Fig. 3A-D). Soft agar assays showed that miR-455-3p depletion markedly increased anchorage-independent growth ability of both LNCaP and PC-3 cells (Fig. 3E and F). We next detected the role of silencing endogenous miR-455-3p on prostate cancer cell tumorigenesis. Nude mice bearing PC-3cell-derived tumors were injected with antagomir-455-3p or negative control through the lateral tail vein every three days for 3 weeks. As shown in Fig. 3E, antagomir-455-3p treatment significantly enhanced prostate cancer cell tumorigenic ability in vivo. Taken together, these results indicate that silencing endogenous miR-455-3p promotes prostate cancer cell growth in vitro and in vivo.

miR-455-3p negatively regulates the expression of eIF4E at the post-transcriptional level by directly binding to the
3'-UTR of eIF4E $m R N A$. To explore the underlying molecular mechanism by which miR-455-3p inhibits prostate cancer cell proliferation, we explored its target genes by employing two bioinformatics tools, TargetScan and miRanda. EIF4E was chosen as a preferred candidate target gene of miR-455-3p due to its role as oncogene in prostate cancer progression and one possible binding site of miR-455-3p in its 3'-untranslated region (3'-UTR) (Fig. 4A). To determine whether EIF4E is a direct target of miR-455-3p, luciferase activity assays were performed with luciferase reporters carrying putative EIF4E 3'-UTR binding sites or mutant binding sites downstream of a luciferase reporter gene (Fig. 4A). As shown in Fig. 4B, miR455-3p overexpression decreased luciferase activity in both LNCaP and PC-3 cells, whereas this suppressive effects was abolished by the potential miR-455-3p binding site mutation in the 3'-UTRs of EIF4E, indicating that miR-455-3p directly targets EIF4E. The opposite result was observed in cells transfected with antagomir-455-3p (Fig. 4C), suggesting that the 3'-UTRs of EIF4E was suppressed by endogenous miR-455-3p. Furthermore, western blot assays showed that miR-455-3p overexpression suppressed the protein expression level of EIF4E in both LNCaP and PC-3 cells, whereas miR-455-3p inhibition promoted its protein expression level (Fig. 4E and G). However, miR-455-3p expression has little effect on the mRNA level of EIF4E (Fig. 4D and F). Taken together, these results indicate that miR-455-3p directly targets the EIF4E 3'-UTR to suppress it protein expression at the post-transcriptional level. 
A

\begin{tabular}{|c|c|}
\hline eIF4E Mut & $5^{\prime} \ldots$ CUUCAAAAAGCAGAG $\underline{A C C U G A C \mathrm{C} . . .}$ \\
\hline eIF4E WT & $5^{\prime} \ldots$ CUUCAAAAAGCAGAG $\frac{U G G A C U G C . .}{|||||| \mid}$ \\
\hline & CACAUAUACGGGUACCUGACG \\
\hline
\end{tabular}

B

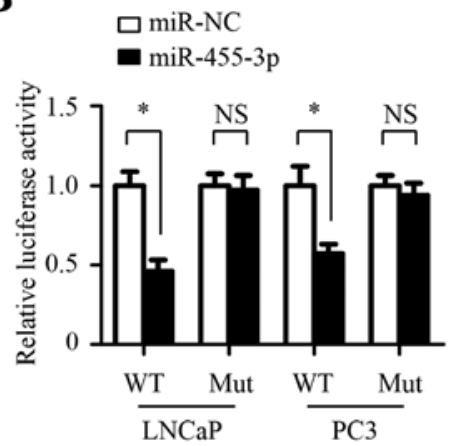

D

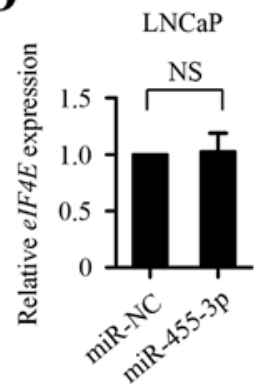

F

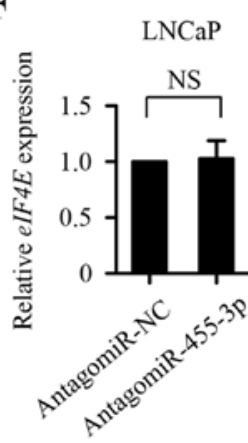

C

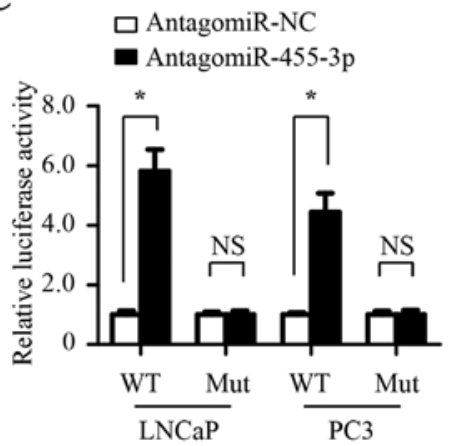

$\mathbf{E}$

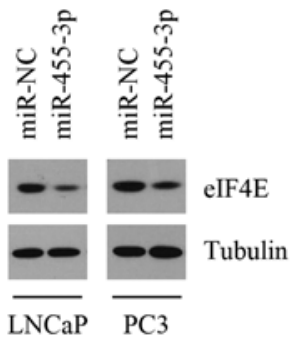

G

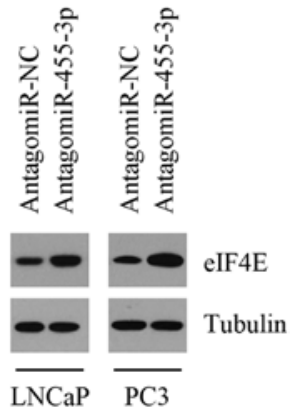

Figure 4. miR-455-3p suppresses eIF4E expression by directly targeting its 3' untranslated region. (A) The target sites of miR-455-3p in 3'-UTR of EIF4E mRNA are shown as a schematic representation. (B) Luciferase reporter assay. Wild type or mutant 3'-UTR construct of EIF4E were cloned into pMIR-Reporter plasmid, respectively, and co-transfected with miR-NC or miR-455-3p to LNCaP and PC-3 cells. Luciferase activities were measured after $48 \mathrm{~h}$. Results are shown as means $\pm \mathrm{SD}$ of triplicate measurements. ${ }^{*} \mathrm{P}<0.05$; NS, not significant. (C) Luciferase reporter assay. pMIR-Reporter plasmid carrying wild-type or mutant eIF4E 3'-UTR was transfected into LNCaP and PC-3 cells along with either antagomir-NC or antagomir-455-3p and analyzed for luciferase activity after $48 \mathrm{~h}$. Results are shown as means \pm SD of triplicate measurements. "P<0.05; NS, not significant. (D and F) q-PCR analysis of eIF4E mRNA levels in LNCaP and PC-3 cells transfected with miR-NC or miR-455-3p (D), antagomir-NC or antagomir-455-3p (F). Expression of eIF4E mRNA was normalized to ACTIN. Results are shown as means \pm SD of triplicate measurements. NS, not significant. (E and G) Western blot analysis of eIF4E protein levels in LNCaP and PC-3 cells transfected with miR-NC or miR-455-3p (E), antagomir-NC or antagomir-455-3p (G). Tubulin was used as a loading control.

miR-455-3p inhibits cap-dependent translation in prostate cancer cells. Given that the expression level of eIF4E is the rate-limiting factor for the assembly of the eIF4F complex and cap-dependent translation and we have established the inhibitory role of miR-455-3p on the expression of eIF4E, we presumed that miR-455-3p may suppress cap-dependent translation in prostate cancer cells. To test this hypothesis, the cap-dependent translation rate in prostate cancer cells transfected with miR-455-3p mimics or antagomir-455-3p was determined using a dual-luciferase reporter system which can monitor the ratio between cap-dependent (Renilla luciferase) and cap-independent IRES-mediated (firefly luciferase) translation initiation. As shown in Fig. 5A-Da, miR-455-3p overexpression inhibited cap-dependent translation in both LNCaP and PC-3 cells, whereas miR-455-3p inhibition promoted cap-dependent translation. We next analyzed the effect of miR-455-3p on the expression of cyclin D1 and c-MYC, two well-known oncogenic genes regulated by capdependent translation. As shown in Fig. 5E and G, miR-455-3p overexpression led to decreased protein levels of cyclin D1 and 
A

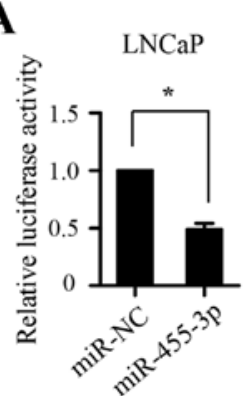

B

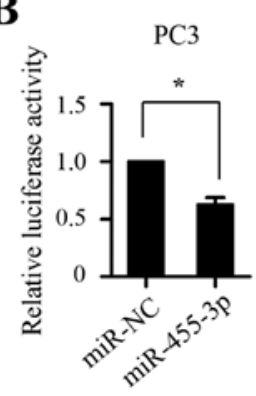

C

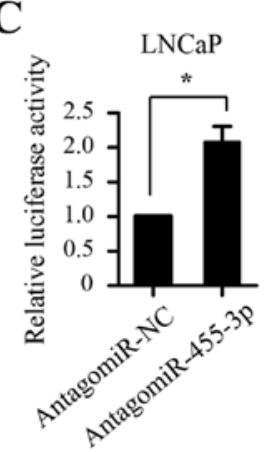

D a

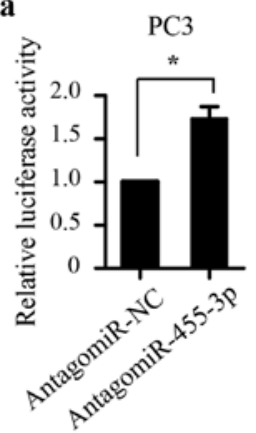

D b

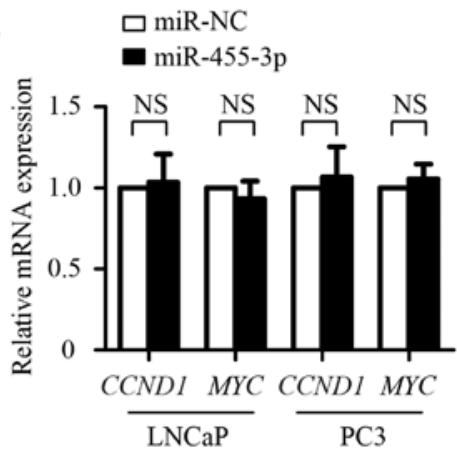

F

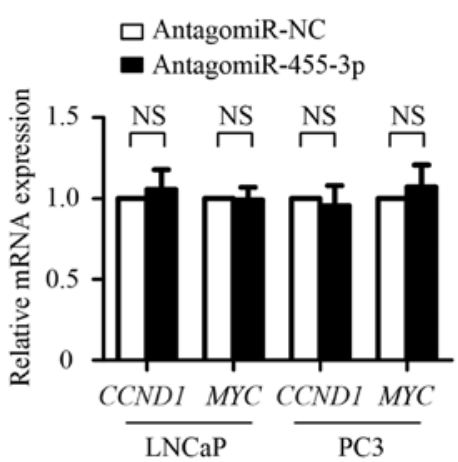

E

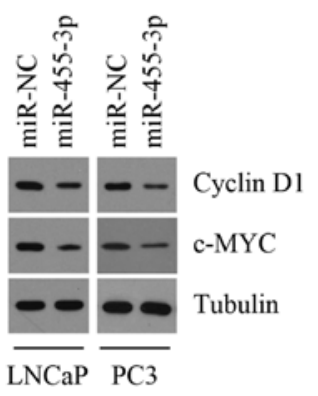

G

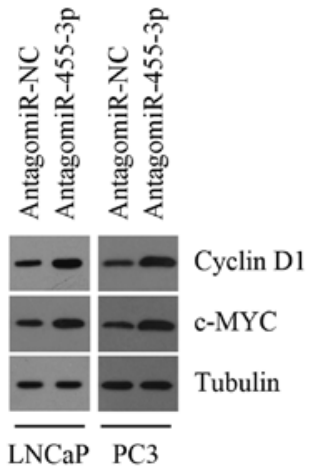

Figure 5. miR-455-3p inhibits cap-dependent translation in prostate cancer cells. (A and B) Bicistronic luciferase assays. A bicistronic luciferase reporter plasmid co-transfected with miR-NC or miR-455-3p to LNCaP (A) and PC-3 cells (B). Luciferase activities were determined after 48 h. Cap-dependent translational activity was determined by calculating the ratio of Renilla/firefly luciferase luminescence. Results are shown as means \pm SD of triplicate measurements. ${ }^{*} \mathrm{P}<0.05$. (C and Da) Bicistronic luciferase assays. A bicistronic luciferase reporter plasmid was transfected into LNCaP and PC-3 cells along with either antagomir-NC or antagomir-455-3p and analyzed for luciferase activity after $48 \mathrm{~h}$. Results are shown as means \pm SD of triplicate measurements. ${ }^{*} \mathrm{P}<0.05$. (Db and F) q-PCR analysis of $C C N D 1$ and $M Y C$ mRNA levels in LNCaP and PC-3 cells transfected with miR-NC or miR-455-3p (Db), antagomir-NC or antagomir-455-3p (F). Expression of CCND1 and MYC mRNA was normalized to ACTIN. Results are shown as means \pm SD of triplicate measurements. NS, not significant. (E and G) Western blot analysis of cyclin D1 and c-MYC protein levels in LNCaP and PC-3 cells transfected with miR-NC or miR-455-3p (E), antagomir-NC or antagomir-455-3p (G). Tubulin was used as a loading control.

c-MYC in LNCaP and PC-3 cells, whereas miR-455-3p inhibition resulted in increased protein levels of c-MYC. Contrary to the inhibition of cyclin D1 and c-MYC at the protein level by miR-455-3p mimics and the increase by antagomir-455-3p, the miR-455-3p mimics or antagomir-455-3p have little effect on the mRNA levels of $C C N D 1$ and $M Y C$ (Fig. 5Db and F), suggesting that the inhibitory effect of cyclin D1 and c-MYC expression by miR-455-3p results from the repression of their translational inhibition rather than that of their gene transcription. Taken together, our results suggest that miR-455-3p inhibits cap-dependent translation in prostate cancer cells.

miR-455-3p inhibits cap-dependent translation and the proliferation of prostate cancer cells through targeting EIF4E. To explore the functional significance of EIF4E in cap-dependent translation and the proliferation of prostate cancer cells suppressed by miR-455-3p, 3' UTR-deleted eIF4E plasmid was introduced into PC-3 cells transfected with miR-455-3p mimics, and then cap-dependent translation and cell proliferation was determined by dual-luciferase reporter system and CCK8 assays, respectively. As shown in Fig. 6A-C, ectopic overexpression of miR-455-3p resulted in reduced cap-dependent translation rates and suppressed proliferation of PC-3 cells, whereas co-transfection with eIF4E plasmid reversed inhibition of cap-dependent translation and cell proliferation caused by miR-455-3p mimics. We next analyzed the effect of eIF4E silencing on antagomir-455-3p-mediated promotion of cap-dependent translation and cell proliferation of PC-3 cells. As shown in Fig. 6D-F, PC-3 cells transfected with antagomir-455-3p exhibited markedly increased capdependent translation rates and cell proliferation, whereas silencing eIF4E expression markedly abrogated these increase. 
A

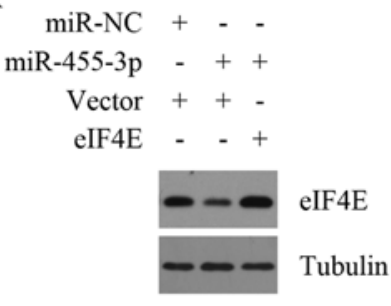

B

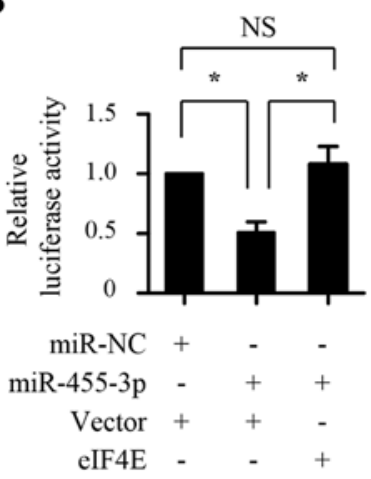

C

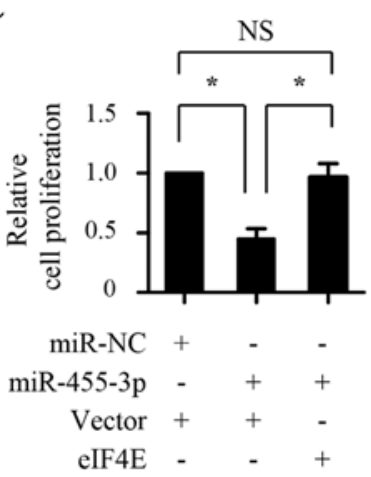

D

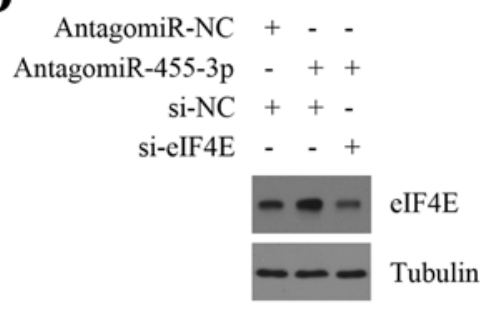

$\mathbf{E}$

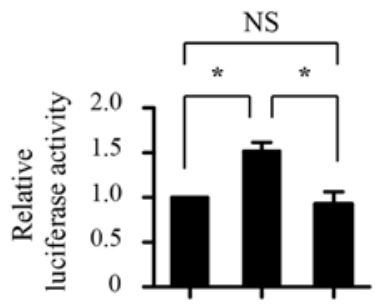

AntagomiR-NC + - -

AntagomiR-455-3p - + +

si-NC ++ -

$\mathbf{F}$

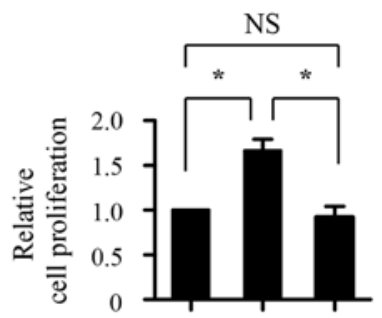

AntagomiR-NC + - -

AntagomiR-455-3p - ++

$\mathrm{si}-\mathrm{NC}++$

Figure 6. miR-455-3p inhibits cap-dependent translation and the proliferation of prostate cancer cells through targeting eIF4E. (A) Western blot analysis of eIF4E expression in PC3 cells co-transfected with miR-NC + pcDNA3.1 vector, miR-455-3p + pcDNA3.1 vector or miR-455-3p + pcDNA3.1-eIF4E. Tubulin was used as a loading control. (B and C) Bicistronic luciferase assay (B) and CCK8 assay (C) of PC3 cells in indicated groups. Results are shown as means \pm SD of triplicate measurements. ${ }^{*} \mathrm{P}<0.05$; NS, not significant. (D) PC3 cells were co-transfected with antagomir-NC or antagomir-455-3p and the control or eIF4E siRNA. Cells were harvested for western blot analysis after $48 \mathrm{~h}$. Tubulin was used as a loading control. (E) PC3 cells transfected with antagomir-NC or antagomir-455-3p and the control or eIF4E siRNA were co-introduced with a bicistronic luciferase reporter plasmid. After 48 h, the reporter activity was measured using luciferase assays. Results are shown as means \pm SD of triplicate measurements. ${ }^{*} \mathrm{P}<0.05$; NS, not significant. (F) CCK 8 assay of PC3 cells transfected with antagomir-NC or antagomir-455-3p and the control or eIF4E siRNA. Results are shown as means \pm SD of triplicate measurements. ${ }^{*} \mathrm{P}<0.05$; NS, not significant.

Taken together, these results suggest that miR-455-3p inhibits cap-dependent translation and the proliferation of prostate cancer cells through targeting EIF4E.

\section{Discussion}

Growing evidence indicates that miRNAs exhibit altered expression levels in many cancers and are emerging as key regulators in the occurrence and progression of cancer. Therefore, miRNAs have been extensively investigated in cancer research for identifying novel diagnostic and prognostic biomarkers and therapeutic targets. In prostate cancer, many miRNAs have been demonstrated to be aberrantly expressed in prostate cancer tissues and exhibit oncogenic function or tumor suppressor role in prostate tumorigenesis. For example, miR-21 has been shown to be overexpressed in prostate cancer tissues and promote both hormone-dependent and hormone-independent tumor growth of prostate cancer cells (14). Further studies demonstrated that miR-21 promotes the survival and invasion of prostate cancer cells by directly targeting MARCKS and RECK $(23,24)$. It was reported that miRNA-302a expression is significantly downregulated in prostate cancer tissue compared with normal prostate tissue and its overexpression markedly inhibits prostate cancer growth in vitro and in vivo. Moreover, AKT was identified as the direct target gene through which miRNA-302a exerts tumor suppressor role in prostate carcinogenesis (19).

Recent studies demonstrate that miR-455-3p is involved in many physiological and pathological processes. miR455-3p has been identified as an important regulator of brown adipogenesis and promotes the brown adipogenic program and mitochondrial biogenesis by targeting HIF1an-AMPKPGC1a signaling network (25). It was demonstrated that miR-455-3p activates early chondrogenic differentiation by 
directly targeting Runx2 (26). In addition, miR-455-3p was showed to be significantly downregulated in placentas from preeclampsia patients and be linked to aberrant hypoxia signaling in preeclampsia (27). Compared with normal tissues, miR-455-3p was demonstrated to be aberrantly expressed in various cancer tissues, including hepatocellular carcinoma (20), melanoma (21) and thyroid carcinoma (22). However, little is known about the miR-455-3p status in prostate cancer tissues and the possible roles in prostate carcinogenesis. In the present study, we determined the expression of miR-455-3p in prostate cancer tissues and found that its expression in prostate cancer tissues was profoundly decreased compared with that in normal prostate tissues, indicating that miR-455-3p is associated with prostate carcinogenesis and it may function as a tumor suppressor. Further gain-of-function and loss-of-function results reveal that miR-455-3p suppresses prostate cancer cell growth in vitro and in vivo. All these studies demonstrate that miR-455-3p exerts tumor suppressor role in prostate cancer progression.

Although the oncogenic role of eIF4E has been well characterized, the mechanisms by which the eIF4E expression is regulated remain incompletely defined. c-MYC, a wellknown oncogene, has been reported to increase the eIF4E expression at transcriptional level (28). HSP27 enhances eIF4E protein stability by decreasing eIF4E ubiquitination and proteasomal degradation, thus promoting the development of castration resistant prostate cancer (29). Recent studies demonstrate that miRNAs play an important role in the regulation of eIF4E expression. miR-34c-3p was reported to be downregulated in non-small cell lung cancer (NSCLC) tissues and to inhibit NSCLC cell proliferation and migration by targeting eIF4E (30). It was reported that repression of microRNA-768-3p by MEK/ERK signaling enhances the eIF4E expression and protein synthesis in melanoma cells (31). miR-141 was shown to repress the eIF4E expression and confer docetaxel chemoresistance of breast cancer cells (32).

Using a database search, we identified that the 3'-UTR of EIF4E mRNA contains a putative miR-455-3p binding site. Reporter assay and western blotting demonstrated that miR455-3p negatively regulates the expression of EIF4E at the post-transcriptional level by directly binding to the 3'-UTR of EIF4E mRNA. EIF4E, as a component of the eIF4F complex, determines the cap-dependent translation activity and plays an important role in tumorigenesis through controlling the translation initiation of a wide range of proteins involving hallmarks of cancers, such as cell cycle, survival, migration and angiogenesis. In agreement with its critical role in tumorigenesis, elevated expression of eIF4E has been shown in prostate cancer patient tissues and eIF4E depletion inhibits prostate cancer cell growth in vitro and in vivo $(9,10)$. Given that we have found that miR-455-3p suppresses the expression of eIF4E in prostate cancer cells, we next determined the effect of miR-455-3p on the cap-dependent translation activity. Using bicistronic luciferase reporter assays, we found that miR-455-3p suppresses the cap-dependent translation activity in prostate cancer cells.

Further results demonstrate that the inhibitory effect of miR-455-3p mimics on cap-dependent translation and cell proliferation in prostate cancer cells can be effectively reversed by eIF4E overexpression, indicating the functional significance of EIF4E in mediating the tumor suppressor role of miR-455-3p. Taken together, our results suggest miR455-3p inhibits cap-dependent translation and the proliferation of prostate cancer cells through targeting eIF4E. Whether the expression level of miR-455-3p is negatively associated with that of eIF4E in clinical tissues remains to be addressed and is a research direction we are currently pursuing. It has been reported that eIF4E shows a higher expression in advanced prostate cancer tissues when compared with low-grade prostate cancer tissues, and promote the development of castration resistant prostate cancer (CRPC) $(29,33)$. Therefore, it will be interesting to determine whether miR-455-3p is involved in CRPC progression by targeting eIF4E in a future study.

In summary, in this study we present evidence that miR-455-3p acts as a tumor suppressor by limiting eIF4E expression in prostate cancer progression. miR-455-3p was markedly downregulated in prostate cancer tissues and suppressed prostate cancer cell growth in vitro and in vivo. Although further studies are required to determine the mechanism by which the expression of miR-455-3p is repressed in prostate cancer, our results indicate that miR-455-3p plays a critical role in prostate malignant transformation and may serve as a novel therapy target for prostate cancer treatment.

\section{Acknowledgements}

This work was supported by Baotou Medical and Health Fund (WSIJ2014038 to H.J.).

\section{References}

1. Siegel RL, Miller KD and Jemal A: Cancer statistics, 2016. CA Cancer J Clin 66: 7-30, 2016.

2. Chen W, Zheng R, Baade PD, Zhang S, Zeng H, Bray F, Jemal A, $\mathrm{Yu}$ XQ and He J: Cancer statistics in China, 2015. CA Cancer J Clin 66: 115-132, 2016.

3. Watson PA, Arora VK and Sawyers CL: Emerging mechanisms of resistance to androgen receptor inhibitors in prostate cancer. Nat Rev Cancer 15: 701-711, 2015.

4. Pettersson F, Del Rincon SV and Miller WH Jr: Eukaryotic translation initiation factor $4 \mathrm{E}$ as a novel therapeutic target in hematological malignancies and beyond. Expert Opin Ther Targets 18: 1035-1048, 2014.

5. Spilka R, Ernst C, Mehta AK and Haybaeck J: Eukaryotic translation initiation factors in cancer development and progression. Cancer Lett 340: 9-21, 2013.

6. Pelletier J, Graff J, Ruggero D and Sonenberg N: Targeting the eIF4F translation initiation complex: A critical nexus for cancer development. Cancer Res 75: 250-263, 2015.

7. Siddiqui $N$ and Sonenberg N: Signalling to eIF4E in cancer. Biochem Soc Trans 43: 763-772, 2015.

8. Bitting RL and Armstrong AJ: Targeting the PI3K/Akt/mTOR pathway in castration-resistant prostate cancer. Endocr Relat Cancer 20: R83-R99, 2013.

9. Kremer CL, Klein RR, Mendelson J, Browne W, Samadzedeh LK, Vanpatten K, Highstrom L, Pestano GA and Nagle RB: Expression of mTOR signaling pathway markers in prostate cancer progression. Prostate 66: 1203-1212, 2006.

10. Graff JR, Konicek BW, Vincent TM, Lynch RL, Monteith D, Weir SN, Schwier P, Capen A, Goode RL, Dowless MS, et al: Therapeutic suppression of translation initiation factor eIF4E expression reduces tumor growth without toxicity. J Clin Invest 117: 2638-2648, 2007.

11. Mao B and Wang G: MicroRNAs involved with hepatocellular carcinoma (Review). Oncol Rep 34: 2811-2820, 2015.

12. Gambari R, Brognara E, Spandidos DA and Fabbri E: Targeting oncomiRNAs and mimicking tumor suppressor miRNAs: New trends in the development of miRNA therapeutic strategies in oncology (Review). Int J Oncol 49: 5-32, 2016. 
13. Garzon R and Marcucci G: Potential of microRNAs for cancer diagnostics, prognostication and therapy. Curr Opin Oncol 24: 655-659, 2012.

14. Ribas J, Ni X, Haffner M, Wentzel EA, Salmasi AH, Chowdhury WH, Kudrolli TA, Yegnasubramanian S, Luo J Rodriguez R, et al: miR-21: An androgen receptor-regulated microRNA that promotes hormone-dependent and hormoneindependent prostate cancer growth. Cancer Res 69: 7165-7169, 2009.

15. Eberlein C, Kendrew J, McDaid K, Alfred A, Kang JS, Jacobs VN, Ross SJ, Rooney C, Smith NR, Rinkenberger J, et al: A human monoclonal antibody 264RAD targeting av $\beta 6$ integrin reduces tumour growth and metastasis, and modulates key biomarkers in vivo. Oncogene 32: 4406-4416, 2013.

16. Fendler A, Jung M, Stephan C, Erbersdobler A, Jung K and Yousef GM: The antiapoptotic function of miR-96 in prostate cancer by inhibition of FOXO1. PLoS One 8: e80807, 2013.

17. Qu F, Cui X, Hong Y, Wang J, Li Y, Chen L, Liu Y, Gao Y, Xu D and Wang Q: MicroRNA-185 suppresses proliferation, invasion, migration, and tumorigenicity of human prostate cancer cells through targeting androgen receptor. Mol Cell Biochem 377: $121-130,2013$.

18. Zhang GM, Bao CY, Wan FN, Cao DL, Qin XJ, Zhang HL, Zhu Y, Dai B, Shi GH and Ye DW: MicroRNA-302a suppresses tumor cell proliferation by inhibiting AKT in prostate cancer PLoS One 10: e0124410, 2015.

19. Huang K, Tang Y, He L and Dai Y: MicroRNA-340 inhibits prostate cancer cell proliferation and metastasis by targeting the MDM2-p53 pathway. Oncol Rep 35: 887-895, 2016.

20. Shubbar E, Kovács A, Hajizadeh S, Parris TZ, Nemes S, Gunnarsdóttir K, Einbeigi Z, Karlsson P and Helou K: Elevated cyclin $\mathrm{B} 2$ expression in invasive breast carcinoma is associated with unfavorable clinical outcome. BMC Cancer 13: 1, 2013.

21. Segura MF, Belitskaya-Lévy I, Rose AE, Zakrzewski J, Gaziel A, Hanniford D, Darvishian F, Berman RS, Shapiro RL, Pavlick AC, et al: Melanoma microRNA signature predicts post-recurrence survival. Clin Cancer Res 16: 1577-1586, 2010.

22. Swierniak M, Wojcicka A, Czetwertynska M, Stachlewska E, Maciag M, Wiechno W, Gornicka B, Bogdanska M, Koperski L, de la Chapelle A, et al: In-depth characterization of the microRNA transcriptome in normal thyroid and papillary thyroid carcinoma. J Clin Endocrinol Metab 98: E1401-E1409, 2013.

23. Li T, Li D, Sha J, Sun P and Huang Y: MicroRNA-21 directly targets MARCKS and promotes apoptosis resistance and invasion in prostate cancer cells. Biochem Biophys Res Commun 383: $280-285,2009$.
24. Reis ST, Pontes-Junior J, Antunes AA, Dall'Oglio MF, Dip N, Passerotti CC, Rossini GA, Morais DR, Nesrallah AJ, Piantino C, et al: miR-21 may acts as an oncomir by targeting RECK, a matrix metalloproteinase regulator, in prostate cancer. BMC Urol 12: 14, 2012.

25. Zhang H, Guan M, Townsend KL, Huang TL, An D, Yan X, Xue R, Schulz TJ, Winnay J, Mori M, et al: MicroRNA-455 regulates brown adipogenesis via a novel HIFlan-AMPK-PGC1 $\alpha$ signaling network. EMBO Rep 16: 1378-1393, 2015.

26. Zhang Z, Hou C, Meng F, Zhao X, Zhang Z, Huang G, Chen W, Fu M and Liao W: MiR-455-3p regulates early chondrogenic differentiation via inhibiting Runx2. FEBS Lett 589: 3671-3678, 2015.

27. Lalevée $\mathrm{S}$, Lapaire $\mathrm{O}$ and Bühler M: miR455 is linked to hypoxia signaling and is deregulated in preeclampsia. Cell Death Dis 5: e1408, 2014.

28. Rosenwald IB, Rhoads DB, Callanan LD, Isselbacher KJ and Schmidt EV: Increased expression of eukaryotic translation initiation factors eIF-4E and eIF-2 alpha in response to growth induction by c-myc. Proc Natl Acad Sci USA 90: 6175-6178, 1993.

29. Andrieu C, Taieb D, Baylot V, Ettinger S, Soubeyran P, De-Thonel A, Nelson C, Garrido C, So A, Fazli L, et al: Heat shock protein 27 confers resistance to androgen ablation and chemotherapy in prostate cancer cells through eIF4E. Oncogene 29: 1883-1896, 2010.

30. Liu F, Wang X, Li J, Gu K, Lv L, Zhang S, Che D, Cao J, Jin S and Yu Y: miR-34c-3p functions as a tumour suppressor by inhibiting eIF4E expression in non-small cell lung cancer. Cell Prolif 48: 582-592, 2015.

31. Jiang CC, Croft A, Tseng HY, Guo ST, Jin L, Hersey $P$ and Zhang XD: Repression of microRNA-768-3p by MEK/ERK signalling contributes to enhanced mRNA translation in human melanoma. Oncogene 33: 2577-2588, 2014.

32. Yao YS, Qiu WS, Yao RY, Zhang Q, Zhuang LK, Zhou F, Sun LB and Yue L: miR-141 confers docetaxel chemoresistance of breast cancer cells via regulation of EIF4E expression. Oncol Rep 33: 2504-2512, 2015

33. Graff JR, Konicek BW, Lynch RL, Dumstorf CA, Dowless MS, McNulty AM, Parsons SH, Brail LH, Colligan BM, Koop JW, et al: eIF4E activation is commonly elevated in advanced human prostate cancers and significantly related to reduced patient survival. Cancer Res 69: 3866-3873, 2009. 